\title{
A vanishing theorem for operators in Fock space
}

\author{
D. Hasler ${ }^{1 *}$ and I. Herbst ${ }^{2 \dagger}$ \\ 1. Institute of Mathematics, University of Munich, \\ D-80333 Munich, Germany \\ 2. Department of Mathematics, University of Virginia, \\ Charlottesville, VA, USA
}

\begin{abstract}
We consider the bosonic Fock space over the Hilbert space of transversal vector fields in three dimensions. This space carries a canonical representation of the group of rotations. For a certain class of operators in Fock space we show that rotation invariance implies the absence of terms which either create or annihilate only a single particle. We outline an application of this result in an operator theoretic renormalization analysis of Hamilton operators, which occur in non-relativistic qed.
\end{abstract}

AMS Subject Classification: $81 \mathrm{~T} 16,81 \mathrm{~T} 10$

Key words: non-relativistic quantum electrodynamics, Fock space, operator theoretic renormalization, symmetry

\section{Introduction}

In this paper we consider the bosonic Fock space over the Hilbert space of transversal vector fields in three dimensions. This space is used in mathematical models of quantized radiation and carries a canonical representation of the group of rotations. We consider a class of operators in this Fock space which arise in a so called operator theoretic renormalization analysis of non-relativistic quantum electrodynamics (qed) [1, 2]. For operators in this class we prove that rotation invariance implies the absence of terms which either create or annihilate only a single particle. This vanishing theorem implies that under a non-degeneracy assumption marginal terms in operator theoretic renormalization are absent. In [6, 7] this property was used to obtain ground state properties in non-relativistic qed, such as analyticity in a minimal coupling constant or differentiability in the fine structure constant.

\footnotetext{
*E-mail: david.hasler@math.lmu.de, on leave from Ludwig Maximilians University

†E-mail: iwh@virginia.edu.
} 
The vanishing theorem was first shown in [6], where the proof relied on a uniqueness result from 22] (Theorem 3.3). The proof in the present paper is self-contained. Since vanishing theorems have far reaching consequences in the context of operator theoretic renormalization, we consider the short and new proof presented in this paper of value to the scientific literature.

In Section 2 we introduce the Fock space and define a canonical representation of the group of rotations in three dimensions. In Theorem 2.3 the vanishing theorem is stated, the main result of this paper. In Subsection 3.1 we introduce the Hamiltonian of nonrelativistic qed and show that it is rotation invariant, with respect to the representation defined in Section 2. In Subsection 3.2, the vanishing theorem is used to derive a corollary, which outlines applications to operator theoretic renormalization.

\section{Model and Statement of Main Result}

We consider the special group of orthogonal matrices in three dimensions

$$
G:=\left\{R \in M_{3}(\mathbb{R}) \mid \operatorname{det} R=1, R^{T}=R^{-1}\right\},
$$

where $M_{3}(\mathbb{R})$ denotes the set of $3 \times 3$ matrices over the real numbers, with the usual topology. As a subset of $M_{3}(\mathbb{R})$ the group $G$ inherits a natural topology.

Definition 2.1. A representation of $G$ (G-representation) is a strongly continuous map $\mathcal{U}: G \rightarrow \mathcal{B}(\mathcal{H})$ to the unitary operators on $\mathcal{H}$, such that

$$
\mathcal{U}\left(R_{1} R_{2}\right)=\mathcal{U}\left(R_{1}\right) \mathcal{U}\left(R_{2}\right), \quad \forall R_{1}, R_{2} \in G .
$$

We shall adopt the following standard conventions. Given a representation $\mathcal{U}$ of $G$ on $\mathcal{H}$, a vector $v \in \mathcal{H}$ is called $\mathcal{U}$-invariant if $\mathcal{U}(R) v=v$ for all $R \in G$. A subspace $V \subset \mathcal{H}$ is called $\mathcal{U}$-invariant if $\mathcal{U}(G) V \subset V$. An operator $T$ in $\mathcal{H}$ with domain $D$ is called $\mathcal{U}$-invariant if $\mathcal{U}(R) D=D$ and $\mathcal{U}(R) T \mathcal{U}(R)^{*}=T$ for all $R \in G$. We will write $G$-invariant instead of $\mathcal{U}$ invariant, if it is clear from the context what the representation is. By rotation invariant we shall always mean $G$-invariant.

The Hilbert space of vector fields $\mathcal{V}=L^{2}\left(\mathbb{R}^{3} ; \mathbb{C}^{3}\right)$ carries a natural representation of $G$, which will be denoted by $\mathcal{U}_{\mathcal{V}}$. Explicitly on vector fields $v \in \mathcal{V}$ it acts as

$$
\left[\mathcal{U}_{\mathcal{V}}(R) v\right](\cdot)=R\left[v\left(R^{-1} \cdot\right)\right],
$$

for all $R \in G$. We define the subspace

$$
\mathfrak{v}:=\left\{v \in \mathcal{V} \mid k \cdot v(k)=0 \text { a.e. } k \in \mathbb{R}^{3}\right\},
$$

of transversal vector fields in $\mathcal{V}$. It is straightforward to verify, that $\mathfrak{v}$ is invariant under the representation $\mathcal{U}_{\mathcal{V}}$. The main result will be based on the following lemma.

Lemma 2.1. If $v \in \mathfrak{v}$ is $G$-invariant, then $v=0$. 
We note that under the simplifying assumption that $v \in \mathfrak{v}$ is defined everywhere and $\left(\mathcal{U}_{\mathfrak{v}}(R) v\right)(k)=v(k)$ for all $k \in \mathbb{R}^{3}$, the assertion of the Lemma follows trivially from the hairy ball theorem. To this end, observe that for such a $v \in \mathfrak{v}$

$$
v(r R e)=R v(r e)
$$

for all $R \in G, r \geq 0$, and $e \in S_{2}:=\left\{e \in \mathbb{R}^{3}|| e \mid=1\right\}$. Eq. (2.2) implies that for every $r>0$, the function $e \mapsto v(r e)$ is a continuous function of $e \in S^{2}$ and its value has constant norm. On the other hand $v(r e)$ is tangential to $S^{2}$ and this contradicts the hairy ball theorem unless it is the zero vector. The problem with this argument is that elements of $\mathfrak{v}$ are only defined up to sets of measure zero. To deal with this issue we decompose $\mathfrak{v}$ into the irreducible representations of $G$. The irreducible unitary representations of $G$ are denoted by $D_{j}, j \in \mathbb{N}_{0}$, and are uniquely determined up to unitary equivalence by their dimension $\operatorname{dim} D_{j}=2 j+1$. For the proof of Lemma 2.2 we use the following idea from [6].

Proof of Lemma 2.2. By means of the canonical isomorphism

$$
L^{2}\left(\mathbb{R}_{+} ; r^{2} d r\right) \otimes L^{2}\left(S_{2} ; \mathbb{C}^{3}\right) \cong \mathcal{V}
$$

we can identify

$$
\mathcal{V}_{0}:=L^{2}\left(\mathbb{R}_{+} ; r^{2} d r\right) \otimes \mathcal{S}
$$

where $\mathcal{S}:=\left\{f \in L^{2}\left(S_{2} ; \mathbb{C}^{3}\right) \mid f(e)=\lambda e, \lambda \in \mathbb{C}\right\}$, with a $G$-invariant subspace of $\mathcal{V}$. It follows from (2.1) that each element in $\mathcal{V}_{0}$ is $G$-invariant. On the other hand it is an immediate consequence of the definition of $\mathcal{S}$ that $\mathcal{V}_{0}$ does not contain any nonzero transversal vector fields. The Lemma will follow if we can show that every $G$-invariant element of $\mathcal{V}$ lies in $\mathcal{V}_{0}$. To this end, let $H_{l}$ denote the space of spherical harmonics with angular momentum $l$. Using $L^{2}\left(S_{2} ; \mathbb{C}^{3}\right)=\bigoplus_{l=0}^{\infty} H_{l} \otimes \mathbb{C}^{3}$, we find from (2.3) an isomorphism of Hilbert spaces

$$
\mathcal{V} \cong L^{2}\left(\mathbb{R}_{+} ; r^{2} d r\right) \otimes \bigoplus_{l=0}^{\infty}\left\{H_{l} \otimes \mathbb{C}^{3}\right\} .
$$

From (2.1) it follows that $G$ acts on $H_{l} \otimes \mathbb{C}^{3}$ as $D_{l} \otimes D_{1}$. By the Clebsch-Gordon decomposition we see that this representation contains the trivial representation only if $l=1$, in which case $D_{1} \otimes D_{1} \cong D_{2} \oplus D_{1} \oplus D_{0}$. Since the trivial representation only occurs once in $L^{2}\left(S_{2} ; \mathbb{C}^{3}\right)$, it follows that $\mathcal{V}_{0}$ contains all elements in $\mathcal{V}$ which are $G$-invariant.

We choose two Borel-measurable endomorphisms

$$
\varepsilon_{1}, \varepsilon_{2}: S_{2} \rightarrow S_{2}
$$

with the property that for a.e. $e \in S^{2}, \varepsilon_{1}(e) \times \varepsilon_{2}(e)=e$, where $\times$ denotes the vector product. We extend these mappings to the set $\mathbb{R}_{\times}^{3}:=\mathbb{R}^{3} \backslash\{0\}$ by setting $\varepsilon_{\lambda}(k)=\varepsilon_{\lambda}(k /|k|)$ for $\lambda=1,2$ and $k \in \mathbb{R}_{\times}^{3}$. The explicit choice of these so called polarization vectors 
establishes a canonical isomorphism, $\phi$, from the Hilbert space $\mathfrak{h}:=L^{2}\left(\mathbb{R}^{3} \times \mathbb{Z}_{2}\right)$, to the Hilbert space of transversal vector fields, i.e.,

$$
\begin{aligned}
\phi: \mathfrak{h} & \rightarrow \mathfrak{v} \\
h & \mapsto\left\{k \mapsto \varepsilon_{1}(k) h(k, 1)+\varepsilon_{2}(k) h(k, 2)\right\} .
\end{aligned}
$$

By means of this isomorphism we obtain the $G$-representation, $\mathcal{U}_{\mathfrak{h}}$, on $\mathfrak{h}$ as follows

$$
\mathcal{U}_{\mathfrak{h}}(R)=\phi^{-1} \mathcal{U}_{\mathfrak{v}}(R) \phi, \quad \forall R \in G .
$$

From Eq. (2.6) the following Corollary is an immediate consequence of Lemma 2.2.

Corollary 2.2. If $h \in \mathfrak{h}$ is $G$-invariant, then $h=0$.

Next we introduce the bosonic Fock space over the Hilbert space $\mathfrak{h}$. We define

$$
\mathcal{F}:=\bigoplus_{n=0}^{\infty} \mathcal{F}_{n}
$$

with $\mathcal{F}_{0}:=\mathbb{C}$ and $\mathcal{F}_{n}:=S_{n}\left(\mathfrak{h}^{\otimes n}\right)$ for $n \in \mathbb{N}$, where $S_{n}$ denotes the orthogonal projection onto the subspace of totally symmetric tensors in $\mathfrak{h}^{\otimes n}$. The vector $\Omega:=(1,0,0, \ldots) \in \mathcal{F}$ is called the Fock vacuum. The Fock space inherits a natural inner product from the Hilbert space $\mathfrak{h}$. The creation operator, $a^{*}(f)$, for $f \in \mathfrak{h}$ is defined on vectors $\eta \in \mathcal{F}_{n}$, with $n \in \mathbb{N}_{0}$, by

$$
a^{*}(f) \eta:=(n+1)^{1 / 2} S_{n+1}(f \otimes \eta) .
$$

We extend this definition by linearity to a densely defined linear operator on $\mathcal{F}$. The resulting operator is closable and its closure will be denoted by the same symbol. We introduce the annihilation operator by

$$
a(f):=\left\{a^{*}(f)\right\}^{*} .
$$

For a bounded linear operator, $A$, on $\mathfrak{h}$, we denote by $\Gamma(A)$ the unique bounded linear operator on $\mathcal{F}$ satisfying $\Gamma(A) \Omega=\Omega$ and

$$
\Gamma(A) S_{n}\left(\varphi_{1} \otimes \cdots \otimes \varphi_{n}\right)=S_{n}\left(A \varphi_{1} \otimes \cdots \otimes A \varphi_{n}\right),
$$

for any $\varphi_{1}, \ldots, \varphi_{n} \in \mathfrak{h}$ and $n \in \mathbb{N}$. The Fock space $\mathcal{F}$ carries a natural representation of $G$ given by

$$
\mathcal{U}_{\mathcal{F}}:=\Gamma\left(\mathcal{U}_{\mathfrak{h}}\right)
$$

Below we introduce operators on Fock space, which arise in operator theoretic renormalization. First we introduce the operator of the free field energy. We define the function $\omega: \mathbb{R}^{3} \times \mathbb{Z}_{2} \rightarrow \mathbb{R},(k, \lambda) \mapsto|k|$ and denote the corresponding multiplication operator on $\mathfrak{h}$ by the same symbol. The operator of the free field energy, $H_{f}$, is defined as the unique selfadjoint operator on Fock space such that for all $t \in \mathbb{R}$

$$
e^{-i H_{f} t}=\Gamma\left(e^{-i \omega t}\right)
$$


We introduce the sets

$$
B_{1}:=\left\{k \in \mathbb{R}^{3}|| k \mid \leq 1\right\}, \quad X:=B_{1} \times \mathbb{Z}_{2}, \quad I:=[0,1] .
$$

For $m, n \in \mathbb{N}_{0}$, we define the Banach space, $\mathcal{W}_{m, n}$, with norm $\|\cdot\|_{\mathcal{W}_{m, n}}$, defined in (2.15) below, to consist of the measurable functions

$$
w_{m, n}: X^{m} \times X^{n} \rightarrow C^{0}(I ; \mathbb{C}),
$$

satisfying the symmetry property (2.11) and the support property (2.14), below. The symmetry property states that for all $\tilde{K}_{1}, \ldots, \tilde{K}_{m}, K_{1}, \ldots, K_{n} \in X$, one has

$$
w_{m, n}\left(\left(\tilde{K}_{1}, \ldots, \tilde{K}_{m}\right),\left(K_{1}, \ldots, K_{n}\right)\right)=w_{m, n}\left(\left(\tilde{K}_{\tilde{\sigma}(1)}, \ldots, \tilde{K}_{\tilde{\sigma}(m)}\right),\left(K_{\sigma(1)}, \ldots, K_{\sigma(n)}\right)\right),
$$

for any permutation $\sigma$ and $\tilde{\sigma}$ of $\{1, \ldots, n\}$ and $\{1, \ldots, m\}$, respectively. For simplicity of notation we shall write

$$
w_{m, n}\left(r ; K^{(m, n)}\right)=w_{m, n}\left(K^{(m, n)}\right)(r),
$$

for $r \in I$ and $K^{(m, n)} \in X^{m} \times X^{n}$. Moreover, we introduce the following notations

$$
\begin{aligned}
K^{(m, n)} & :=\left(\tilde{K}^{(m)}, K^{(n)}\right):=\left(\left(\tilde{k}_{1}, \tilde{\lambda}_{1}, \ldots, \tilde{k}_{m}, \tilde{\lambda}_{m}\right),\left(k_{1}, \lambda_{1}, \ldots, k_{n}, \lambda_{n}\right)\right) \in X^{m} \times X^{n} \\
\left|K^{(m)}\right| & :=\prod_{j=1}^{m}\left|k_{j}\right|, \quad d K^{(m)}:=\prod_{j=1}^{m} d k_{j}^{3}, \quad \Sigma\left[K^{(n)}\right]:=\sum_{j=1}^{n}\left|k_{j}\right| .
\end{aligned}
$$

The support property states that

$$
w_{m, n}\left(r ; K^{(m, n)}\right)=1_{\Sigma\left[\tilde{K}^{(m)}\right]+r \leq 1} w_{m, n}\left(r ; K^{(m, n)}\right) 1_{\Sigma\left[K^{(n)}\right]+r \leq 1},
$$

for all $r \in I$ and $K^{(m, n)} \in X^{m+n}$. The norm is given by

$$
\left\|w_{m, n}\right\|_{\mathcal{W}_{m, n}}:=\left\{\int_{X^{m} \times X^{n}} \sup _{r \in I}\left|w_{m, n}\left(r ; K^{(m, n)}\right)\right|^{2} d \mu_{m, n}^{(2)}\left(K^{(m, n)}\right)\right\}^{1 / 2},
$$

with measure

$$
d \mu_{m, n}^{(p)}\left(K^{(m, n)}\right):=\frac{d \tilde{K}^{(m)}}{\left|\tilde{K}^{(m)}\right|^{p}} \frac{d K^{(m)}}{\left|K^{(n)}\right|^{p}} .
$$

The integration in (2.15) includes summation over $\lambda_{i}$ and $\tilde{\lambda}_{i}$. For $0<\xi<1$, we define the Banach space $\mathcal{W}_{\xi}$ to consist of sequences $\underline{w}=\left(w_{m, n}\right)_{(m, n) \in \mathbb{N}_{0}^{2}}$ with $w_{m, n} \in \mathcal{W}_{m, n}$ such that the norm

$$
\|\underline{w}\|_{\xi}:=\sum_{m+n \geq 0} \xi^{-(m+n)}\left\|w_{m, n}\right\|_{\mathcal{W}_{m, n}}
$$

is finite. Let $\underline{w} \in \mathcal{W}_{\xi}$. We define the operator

$$
H_{m, n}[\underline{w}]:=\int_{X^{m} \times X^{n}} \prod_{i=1}^{m} a_{\tilde{\lambda}_{i}}^{*}\left(\tilde{k}_{i}\right) w_{m, n}\left(H_{f} ; K^{(m, n)}\right) \prod_{j=1}^{n} a_{\lambda_{j}}\left(k_{j}\right) d \mu_{m, n}^{(1 / 2)}\left(K^{(m, n)}\right) .
$$


Definition (2.17) is understood in the sense of forms and the right hand side of (2.17) as a weak integral. Moreover, the definition involves the operator valued distributions $a_{\lambda}(k)$ and $a_{\lambda}^{*}(k)$ which are characterized by the relations

$$
a^{*}(f)=\sum_{\lambda=1,2} \int f(k, \lambda) a_{\lambda}^{*}(k) d^{3} k, \quad a(f)=\sum_{\lambda=1,2} \int \overline{f(k, \lambda)} a_{\lambda}(k) d^{3} k,
$$

for all $f \in \mathfrak{h}$. In the Appendix we give an explicit and rigorous definition of (2.17) which does not involve any creation or annihilation operators.

Remark 1. In this paper we view $H[\underline{w}]$ as on operator on Fock space. To this end we tacitly extend the kernels $w_{m, n}$ to functions on $\mathbb{R} \times\left\{\mathbb{R}^{3} \times \mathbb{Z}_{2}\right\}^{m+n}$ by setting them equal to zero on the complement of $I \times X^{m+n}$. Alternatively, we could view $H[\underline{w}]$ to be an operator on $1_{H_{f} \leq 1} \mathcal{F}$. This would not change the analysis. By assumption (2.14) we can neglect projection operators $1_{H_{f} \leq 1}$ in the definition (2.17), which appear in [2].

With respect to the operator norm in Fock space the following estimate is shown in the Appendix,

$$
\left\|H_{m, n}[\underline{w}]\right\| \leq\left\|w_{m, n}\right\|_{\mathcal{W}_{m, n}} .
$$

We define the operator

$$
H[\underline{w}]:=\sum_{m+n \geq 0} H_{m, n}[\underline{w}] .
$$

It follows from (2.18) and the definition of the norm (2.16) that the right hand side of (2.19) converges absolutely with respect to the operator norm, and furthermore,

$$
\|H[\underline{w}]\| \leq\|\underline{w}\|_{\xi}
$$

Now we state the main result of this letter.

Theorem 2.3. Let $\underline{w} \in \mathcal{W}_{\xi}$. If $H[\underline{w}]$ is a $G$-invariant operator, then $H_{0,1}[\underline{w}]=0$ and $H_{1,0}[\underline{w}]=0$.

Proof. For $f_{1}, f_{2}, g \in \mathfrak{h}$ it follows from definition (2.17) (or respectively definitions (A.2) and $(\underline{A .3})$ ), that

$$
\begin{aligned}
& \frac{1}{\sqrt{2}}\left\langle g, H[\underline{w}] S_{2}\left(f_{1} \otimes f_{2}\right)\right\rangle= \\
& \quad \frac{1}{2} \sum_{\lambda, \tilde{\lambda}} \int \overline{g(\tilde{k}, \tilde{\lambda})} f_{1}(\tilde{k}, \tilde{\lambda}) w_{0,1}(|\tilde{k}| ; k, \lambda) f_{2}(k, \lambda) \frac{d^{3} \tilde{k} d^{3} k}{\sqrt{|k|}} \\
& \quad+\frac{1}{2} \sum_{\tilde{\lambda}, \lambda} \int \overline{g(\tilde{k}, \tilde{\lambda})} f_{2}(\tilde{k}, \tilde{\lambda}) w_{0,1}(|\tilde{k}| ; k, \lambda) f_{1}(k, \lambda) \frac{d^{3} \tilde{k} d^{3} k}{\sqrt{|k|}} \\
& \quad+\sum_{\tilde{\lambda}, \lambda_{1}, \lambda_{2}} \int \overline{g(\tilde{k}, \tilde{\lambda})} w_{1,2}\left(0 ; \tilde{k}, \tilde{\lambda}, k_{1}, \lambda_{1}, k_{2}, \lambda_{2}\right) f_{1}\left(k_{1}, \lambda_{1}\right) f_{2}\left(k_{2}, \lambda_{2}\right) \frac{d^{3} \tilde{k} \ldots d^{3} k_{2}}{\sqrt{|\tilde{k}|\left|k_{1}\right|\left|k_{2}\right|}} .
\end{aligned}
$$


Pick a function $\varphi \in C_{0}^{\infty}\left(B_{1} ; \mathbb{R}\right)$ with $\int \varphi^{2}(k) d^{3} k=1$, and for $x \in B_{1}^{0}:=\left\{k \in \mathbb{R}^{3}|| k \mid<1\right\}$ define

$$
f_{\epsilon, x}(k, \lambda):=2^{-1 / 4} \epsilon^{-3 / 2} \varphi\left(\epsilon^{-1}(x-k)\right), \quad \epsilon>0 .
$$

Notice that $f_{\epsilon, x}$ converges weakly to zero in $L^{2}$ as $\epsilon \downarrow 0$.

We insert the choice $f_{1}=g=\mathcal{U}_{\mathfrak{h}}(R) f_{\epsilon, x}$ and $f_{2}=\mathcal{U}_{\mathfrak{h}}(R) h$, with $h \in \mathfrak{h}$, into (2.20). We claim that in the limit $\epsilon \downarrow 0$, the terms in lines (2.22) and (2.23) vanish. To this end note that

$$
G_{1}(\tilde{k}, k)=f_{2}(\tilde{k}, \tilde{\lambda}) w_{0,1}(|\tilde{k}|, k, \lambda) / \sqrt{|k|}
$$

and

$$
G_{2}\left(\tilde{k}, k_{1}\right)=\int w_{1,2}\left(0, \tilde{k}, \tilde{\lambda}, k_{1}, \lambda_{1}, k_{2}, \lambda_{2}\right) f_{2}\left(k_{2} \cdot \lambda_{2}\right) \frac{d^{3} k_{2}}{\sqrt{|\tilde{k}|\left|k_{1}\right|\left|k_{2}\right|}}
$$

are kernels of Hilbert-Schmidt operators so by the weak convergence of $g=f_{1}$ to 0 , the terms in lines (2.22) and (2.23) vanish.

An elementary calculation using the definition of the polarization vectors, the group of rotations, and (2.6) shows that

$$
\sum_{\lambda=1,2}\left|\left(\mathcal{U}_{\mathfrak{h}} f_{\epsilon, x}\right)(k, \lambda)\right|=\sum_{\lambda=1,2}\left|f_{\epsilon, x}\left(R^{-1} k, \lambda\right)\right|^{2}, \quad \forall k \in \mathbb{R}^{3} .
$$

Using this and the vanishing of (2.22) and (2.23) in the limit $\epsilon \downarrow 0$, we find

$$
\begin{aligned}
& \lim _{\epsilon \downarrow 0}\left\langle\mathcal{U}_{\mathfrak{h}}(R) f_{\epsilon, x}, H[\underline{w}] S_{2}\left(\mathcal{U}_{\mathfrak{h}}(R) f_{\epsilon, x} \otimes \mathcal{U}_{\mathfrak{h}}(R) h\right)\right\rangle \\
& \quad=\lim _{\epsilon \downarrow 0} \frac{1}{\sqrt{2}} \sum_{\lambda, \tilde{\lambda}} \int\left|f_{\epsilon, x}\left(R^{-1} \tilde{k}, \tilde{\lambda}\right)\right|^{2} w_{0,1}(|\tilde{k}| ; k, \lambda)\left(\mathcal{U}_{\mathfrak{h}}(R) h\right)(k, \lambda) \frac{d^{3} \tilde{k} d^{3} k}{\sqrt{|k|}} .
\end{aligned}
$$

Moreover, using dominated convergence one finds

$$
(\underline{2.28})=\frac{1}{\sqrt{2}} \sum_{\lambda, \tilde{\lambda}} \int \lim _{\epsilon \downarrow 0} I(\epsilon, k, \lambda, \tilde{\lambda}, R, h) d^{3} k,
$$

where we introduced the notation

$$
I(\epsilon, k, \lambda, \tilde{\lambda}, R, h):=\int\left|f_{\epsilon, x}\left(R^{-1} \tilde{k}, \tilde{\lambda}\right)\right|^{2} \frac{w_{0,1}(|\tilde{k}| ; k, \lambda)}{|k|^{1 / 2}}\left(\mathcal{U}_{\mathfrak{h}}(R) h\right)(k, \lambda) d^{3} \tilde{k}
$$

and justified dominated convergence by the estimate

$$
|I(\epsilon, k, \lambda, \tilde{\lambda}, R, h)| \leq \sup _{r \in I}\left|w_{0,1}(r ; k, \lambda)\right|\left|\left(\mathcal{U}_{\mathfrak{h}}(R) h\right)(k, \lambda)\right| / \sqrt{|k|}
$$

and the fact that the r.h.s. of (2.30) is integrable w.r.t. $k$ by the finiteness of $(2.15)$. Using that the square of (2.24) yields a delta sequence and that $I \ni r \mapsto w(r ; k, \lambda)$ is for a.e. $k$ a continuous function we find

$$
\lim _{\epsilon \downarrow 0} I(\epsilon, k, \lambda, \tilde{\lambda}, R, h)=2^{-1 / 2} w_{0,1}(|x| ; k, \lambda)\left(\mathcal{U}_{\mathfrak{h}}(R) h\right)(k, \lambda) /|k|^{1 / 2},
$$


$k$ a.e. (depending on $R$ and $h$ ) (notice that we choose $x$ to lie in the interior because otherwise we would get a factor $1 / 2$ ). Inserting this into (2.29) we find

$$
(2.27)=\sum_{\lambda} \int w_{0,1}(|x| ; k, \lambda)\left(\mathcal{U}_{\mathfrak{h}}(R) h\right)(k, \lambda) \frac{d^{3} k}{|k|^{1 / 2}}
$$

By rotation invariance of $H[w]$ it follows that (2.27) is independent of $R$. In view of (2.32) this implies

$$
\sum_{\lambda} \int w_{0,1}(|x| ; k, \lambda) h(k, \lambda) \frac{d^{3} k}{|k|^{1 / 2}}=\sum_{\lambda} \int w_{0,1}(|x| ; k, \lambda)\left(\mathcal{U}_{\mathfrak{h}}(R) h\right)(k, \lambda) \frac{d^{3} k}{|k|^{1 / 2}}
$$

for all $h \in \mathfrak{h}, R \in G$, and $x \in B_{1}^{0}$. Since the the representation $\mathcal{U}_{\mathfrak{h}}(R)$ is unitary and $h \in \mathfrak{h}$ is arbitrary, Eq. (2.33) implies that

$$
\mathcal{U}_{\mathfrak{h}}(R) w_{0,1}^{(|x|)}=w_{0,1}^{(|x|)}, \quad \forall R \in G,
$$

where we introduced the function $w_{0,1}^{(|x|)}:(k, \lambda) \mapsto w_{0,1}(|x| ; k, \lambda)$ which by the finiteness of (2.15) is an element of $L^{2}\left(\mathbb{R}^{3} \times \mathbb{Z}_{2}\right)$. Now (2.34) and Corollary 2.3 imply that $w_{0,1}^{(|x|)}=0$. Since $x \in B_{1}^{0}$ is arbitrary, we conclude that $w_{0,1}=0$ (the vanishing at the endpoint $|x|=1$ follows from continuity) and hence $H_{0,1}[\underline{w}]=0$. Similarly one shows $H_{1,0}[\underline{w}]=0$.

\section{Application}

\subsection{Nonrelativistic qed}

In this subsection we introduce in (3.1) below the Hamiltonian which describes, in the framework of non-relativistic quantum electrodynamics, an atom consisting of $N$ spinless electrons and a nucleus with infinite mass and point charge $Z=N$. In (3.3) we define a natural $G$-representation on the Hilbert space of the $N$ electrons, which yields a representation on the Hilbert space of the total system (3.4). In Equation (3.8) of Proposition 3.2 it will be shown that, with respect to this representation, the Hamiltonian (3.1) is a $G$-invariant operator.

The Hilbert space of the $N$ electrons is $\mathcal{H}_{\text {at }}:=\bigwedge^{N} L^{2}\left(\mathbb{R}^{3}\right)$, and the Hilbert space of the total system is $\mathcal{H}_{0}:=\mathcal{H}_{\text {at }} \otimes \mathcal{F}$. The Hamiltonian is

$$
H:=\sum_{j=1}^{N}\left(p_{j} \otimes \mathbf{1}-e A\left(x_{j}\right)\right)^{2}+e^{2} V_{C} \otimes \mathbf{1}+\mathbf{1} \otimes H_{f}
$$

where $x_{j} \in \mathbb{R}^{3}$ is the position of the $j$-th electron, and $e$ is the electron's charge.

$$
V_{C}:=\sum_{i<j} \frac{1}{\left|x_{i}-x_{j}\right|}-\sum_{j=1}^{N} \frac{Z}{\left|x_{j}\right|}
$$


with $l=1,2,3$ and $x \in \mathbb{R}^{3}$

$$
A(x):=A^{+}(x)+A^{-}(x), \quad A_{l}^{+}(x):=a^{*}\left(\kappa_{l, x}\right), \quad A_{l}^{-}(x):=a\left(\kappa_{l, x}\right),
$$

and for $(k, \lambda) \in \mathbb{R}^{3} \times \mathbb{Z}_{2}$

$$
\kappa_{l, x}(k, \lambda):=1_{|k| \leq \Lambda} \frac{1}{\sqrt{2|k|}}\left[\varepsilon_{\lambda}(k)\right]_{l} e^{-i k \cdot x} .
$$

In (3.2) the number $\Lambda>0$ serves as an ultraviolet cutoff. The Hamiltonian (3.1) can realized as a selfadjoint operator as follows. One can show that (3.1) defines a semibounded closed form on the natural domain of the operator $\left(\Delta \otimes 1+1 \otimes H_{f}\right)^{1 / 2}$, with $\Delta:=\sum_{j=1}^{n} p_{j}^{2}$. By the second representation theorem this yields a unique self-adjoint operator with domain equal to the natural domain of the operator $\Delta \otimes 1+1 \otimes H_{f}$ (for details see for example [4]).

We define a $G$-representation, $\mathcal{U}_{\text {at }}$, on the Hilbert space $\mathcal{H}_{\text {at }}$ by setting

$$
\left(\mathcal{U}_{\mathrm{at}}(R) \psi\right)\left(x_{1}, \ldots, x_{N}\right):=\psi\left(R^{-1} x_{1}, \ldots, R^{-1} x_{N}\right)
$$

for all $\psi \in \mathcal{H}_{\text {at }}, R \in G$, and $\left(x_{1}, \ldots, x_{N}\right) \in \mathbb{R}^{3 N}$. On $\mathcal{H}_{0}$ we define the tensor representation

$$
\mathcal{U}_{0}:=\mathcal{U}_{\text {at }} \otimes \mathcal{U}_{\mathcal{F}}
$$

The next lemma will be used to show Proposition 3.2 below.

Lemma 3.1. For all $x \in \mathbb{R}^{3}$ and $R \in G$ one has $\mathcal{U}_{\mathfrak{h}}(R) \kappa_{l, x}=\sum_{m=1}^{3} R_{l m}^{-1} \kappa_{m, R x}$ as an identity in $\mathfrak{h}$.

Proof. Using the definitions (2.1), (2.5), (2.6) $\delta_{i j}-\frac{k_{i} k_{j}}{|k|^{2}}$, one finds

$$
\begin{array}{r}
\left(\mathcal{U}_{\mathfrak{h}} \kappa_{l, x}\right)(k, \lambda)=\varepsilon_{\lambda}(k) \cdot R\left(\phi \kappa_{l, x}\right)\left(R^{-1} k\right)=\varepsilon_{\lambda}(k) \cdot R \sum_{\lambda^{\prime}} \varepsilon_{\lambda^{\prime}}\left(R^{-1} k\right) \kappa_{l, x}\left(\lambda^{\prime}, R^{-1} k\right) \\
=\sum_{\lambda^{\prime}} \varepsilon_{\lambda}(k) \cdot R \varepsilon_{\lambda^{\prime}}\left(R^{-1} k\right)\left[\varepsilon_{\lambda^{\prime}}\left(R^{-1} k\right)\right]_{l} e^{-i R^{-1} k \cdot x}=\left[R^{-1} \varepsilon_{\lambda}(k)\right]_{l} e^{-i k \cdot R x} \\
=\sum_{m=1}^{3} R_{l m}^{-1} \kappa_{m, R x}(k, \lambda) .
\end{array}
$$

Using Lemma 3.1 it is now straight forward to prove the following proposition, which in physicists terminology states that the vector potential transforms as a so called "vector field" and that the Hamiltonian transforms as a so called "scalar". 
Proposition 3.2. The following transformation properties hold. For all $R \in G$,

$$
\begin{aligned}
\mathcal{U}_{0}(R) A^{ \pm}\left(x_{j}\right) \mathcal{U}_{0}(R)^{*} & =R^{-1} A^{ \pm}\left(x_{j}\right), \quad \text { on the natural domain of }\left(1 \otimes H_{f}\right)^{1 / 2} \\
\mathcal{U}_{\mathcal{F}}(R) H_{f} \mathcal{U}_{\mathcal{F}}(R)^{*} & =H_{f} \\
\mathcal{U}_{0}(R) H_{g} \mathcal{U}_{0}(R)^{*} & =H_{g}
\end{aligned}
$$

Proof. Let $\underline{x}=\left(x_{1}, \ldots, x_{N}\right)$ and $R \underline{x}=\left(R x_{1}, \ldots, R x_{N}\right)$. For $l=1,2,3$ let $x_{j, l}$ denote $l$-th component of $x_{j}$. Then

$$
\left(\mathcal{U}_{\mathrm{at}}(R)\left[x_{j, l} \psi\right]\right)(\underline{x})=\left[x_{j, l} \psi\right]\left(R^{-1} \underline{x}\right)=\left[R^{-1} x_{j}\right]_{l}\left(\mathcal{U}_{\mathrm{at}}(R) \psi\right)(\underline{x}) .
$$

This yields the transformation property

$$
\mathcal{U}_{\mathrm{at}}(R) x_{j} \mathcal{U}_{\mathrm{at}}(R)^{*}=R^{-1} x_{j} .
$$

Let $\partial_{j, l}$ denote the partial derivative with respect to $x_{j, l}$. Then

$$
\left.\left(\mathcal{U}_{\mathrm{at}}(R)\left[\partial_{j, l} \psi\right]\right)(\underline{x})=\left[\partial_{j, l} \psi\right]\right)\left(R^{-1} \underline{x}\right)=\sum_{k=1}^{3} R_{l k}^{-1} \partial_{j, k}\left[\mathcal{U}_{\mathrm{at}}(R) \psi\right](\underline{x}) .
$$

This yields the transformation property

$$
\mathcal{U}_{\mathrm{at}}(R) p_{j} \mathcal{U}_{\mathrm{at}}(R)^{*}=R^{-1} p_{j} .
$$

By Lemma 3.1, and (3.9), we find

$$
\mathcal{U}_{0}(R) A_{l}^{ \pm}\left(x_{j}\right) \mathcal{U}_{0}(R)^{*}=a^{*}\left(\mathcal{U}_{\mathfrak{h}}(R) \kappa_{l, R^{-1} x_{j}}\right)=\sum_{m=1}^{3} R_{l m}^{-1} A_{m}^{ \pm}\left(x_{j}\right) .
$$

This implies (3.6). Eq. (3.7) can be seen using the definition (2.10) and the fact that $\omega$ only depends on $|k|$. Now (3.7), (3.6), (3.9), and (3.10) imply that Eq. (3.8) holds a priori in the sense of forms, and hence as an operator in $\mathcal{H}_{0}$.

For the application of Theorem 2.3 in the context of operator theoretic renormalization we will need Lemma 3.3, stated below. To this end, we first consider the atomic Hamiltonian

$$
H_{\mathrm{at}}:=\sum_{j=1}^{N} p_{j}^{2}+V_{C}
$$

acting on $\mathcal{H}_{\text {at }}$. It is straight forward to see that $H_{\text {at }}$ is $G$-invariant. It is well known that the infimum of the spectrum, $E_{\text {at }}:=\inf \sigma\left(H_{\text {at }}\right)$, is an eigenvalue. We will need the following rather restrictive Hypothesis.

(H) $E_{\text {at }}$ is a non-degenerate eigenvalue of $H_{\text {at }}$.

Now suppose Hypothesis $(\mathbf{H})$ holds. We will denote by $\varphi_{\text {at }}$ the normalized eigenstate of $H_{\text {at }}$ with eigenvalue $E_{\text {at }}$. By hypothesis it follows that $\varphi_{\text {at }}$ is rotation invariant, since one 
dimensional representations of $G$ are trivial. We introduce the projection $P_{\text {at }}:=\left|\varphi_{\text {at }}\right\rangle\left\langle\varphi_{\text {at }}\right|$ in $\mathcal{H}_{\text {at }}$ and set $P:=P_{\text {at }} \otimes \mathbf{1}$. For later use we define the map

$$
V_{\text {at }}: \mathcal{F} \rightarrow \operatorname{Ran} P, \quad \eta \mapsto \varphi_{\text {at }} \otimes \eta
$$

It follows immediately from properties of the tensor product that $V_{\text {at }}$ is unitary.

Lemma 3.3. Suppose $(\mathbf{H})$ holds. Let $T$ be a bounded $G$-invariant operator on $\mathcal{H}_{0}$. Then $V_{\text {at }}^{*} P T P V_{\text {at }}$ is a $G$-invariant operator on $\mathcal{F}$.

Proof. For any $\eta_{1}, \eta_{2} \in \mathcal{F}$ and $R \in G$,

$$
\begin{array}{r}
\left\langle\eta_{1}, V_{\mathrm{at}}^{*} P T P V_{\mathrm{at}} \eta_{2}\right\rangle=\left\langle\varphi_{\mathrm{at}} \otimes \eta_{1}, T\left[\varphi_{\mathrm{at}} \otimes \eta_{2}\right]\right\rangle=\left\langle\varphi_{\mathrm{at}} \otimes \eta_{1}, \mathcal{U}_{0}(R) T \mathcal{U}_{0}(R)^{*}\left[\varphi_{\mathrm{at}} \otimes \eta_{2}\right]\right\rangle \\
=\left\langle\varphi_{\mathrm{at}} \otimes\left(\mathcal{U}_{\mathcal{F}}(R)^{*} \eta_{1}\right), T\left[\varphi_{\mathrm{at}} \otimes\left(\mathcal{U}_{\mathcal{F}}(R)^{*} \eta_{2}\right)\right]\right\rangle=\left\langle\mathcal{U}_{\mathcal{F}}(R)^{*} \eta_{1}, V_{\mathrm{at}}^{*} P T P V_{\mathrm{at}} \mathcal{U}_{\mathcal{F}}(R)^{*} \eta_{2}\right\rangle \\
=\left\langle\eta_{1}, \mathcal{U}_{\mathcal{F}}(R) V_{\mathrm{at}}^{*} P T P V_{\mathrm{at}} \mathcal{U}_{\mathcal{F}}(R)^{*} \eta_{2}\right\rangle
\end{array}
$$

where we used the rotation invariance of $\varphi_{\text {at }}$. Since $\eta_{1}, \eta_{2}$ are arbitrary, the Lemma follows.

\subsection{Smooth Feshbach}

In this subsection we first introduce the so called Feshbach operator, and then state in Corollary 3.4, below, the main application of Theorem 2.3. This Corollary can be used in operator theoretic renormalization to show that marginal terms are absent [6].

Let $\chi$ and $\bar{\chi}$ be commuting, nonzero bounded operators, acting on a separable Hilbert space $\mathcal{H}$ and satisfying $\chi^{2}+\bar{\chi}^{2}=1$. A Feshbach pair $(H, T)$ for $\chi$ is a pair of closed operators with the same domain,

$$
H, T: D(H)=D(T) \subset \mathcal{H} \rightarrow \mathcal{H}
$$

such that $H, T, W:=H-T$, and the operators

$$
\begin{array}{ll}
W_{\chi}:=\chi W \chi, & W_{\bar{\chi}}:=\bar{\chi} W \bar{\chi} \\
H_{\chi}:=T+W_{\chi}, & H_{\bar{\chi}}:=T+W_{\bar{\chi}},
\end{array}
$$

defined on $D(T)$ satisfy the following assumptions:

(a) $\chi T \subset T \chi$ and $\bar{\chi} T \subset T \bar{\chi}$,

(b) $H_{\bar{\chi}}, T: D(T) \cap \operatorname{Ran} \bar{\chi} \rightarrow \operatorname{Ran} \bar{\chi}$ are bijections with bounded inverse,

(c) $\bar{\chi} H_{\bar{\chi}}^{-1} \bar{\chi} W \chi: D(T) \subset \mathcal{H} \rightarrow \mathcal{H}$ is a bounded operator.

Here we used the notation $H_{\bar{\chi}}^{-1} \bar{\chi}:=\left(H_{\bar{\chi}} \uparrow \operatorname{Ran} \bar{\chi}\right)^{-1} \bar{\chi}$. Given a Feshbach pair $(H, T)$ for $\chi$, the operator

$$
F_{\chi}(H, T):=H_{\chi}-\chi W \bar{\chi} H_{\bar{\chi}}^{-1} \bar{\chi} W \chi
$$


is called Feshbach operator. In [3] it is shown that the full spectral information of the original operator $H$ can be recovered by the restriction of the Feshbach operator to any closed subspace $V$ with the property that $\operatorname{Ran} \chi \subset V \subset \mathcal{H}$ and that $\chi V \subset V$.

In operator theoretic renormalization one typically chooses the following realization for the operators $\chi$ and $\bar{\chi}$. Let $\eta \in C([0,1] ;[0,1])$ be a function such that there exist two numbers $a$ and $b$ with $0<a<b<1,\left.\eta\right|_{[0, a]}=1$, and $\left.\eta\right|_{[b, 1]}=0$. Setting $\bar{\eta}:=\left(1-\eta^{2}\right)^{1 / 2}$ it follows that $\eta^{2}+\bar{\eta}^{2}=1$. The operators $\boldsymbol{\chi}:=P_{\mathrm{at}} \otimes \eta\left(H_{f}\right)$ and $\overline{\boldsymbol{\chi}}:=\left(\mathbf{1}-P_{\mathrm{at}}\right) \otimes \mathbf{1}+P_{\mathrm{at}} \otimes \bar{\eta}\left(H_{f}\right)$ satisfy

$$
\chi^{2}+\bar{\chi}^{2}=1
$$

Corollary 3.4. The following statements hold.

(a) Fix $z, g \in \mathbb{C}$. Suppose Hypothesis $(\mathbf{H})$ holds and that $\left(H_{g}-z, H_{0}-z\right)$ is a Feshbach pair for $\chi$. Then the operator $F_{z, g}:=V_{\mathrm{at}}^{*} P F_{\chi}\left(H_{g}-z, H_{0}-z\right) P V_{\mathrm{at}}$ is invariant under rotations. If there exists a $\underline{w}_{z, g} \in \mathcal{W}_{\xi}$ such that $F_{z, g}=H\left[\underline{w}_{z, g}\right]$, then $H_{0,1}\left[\underline{w}_{z, g}\right]=$ $H_{0,1}\left[\underline{w}_{z, g}\right]=0$.

(b) Suppose $\chi=\eta\left(H_{f}\right)$ and $(H, T)$ is a Feshbach pair for $\chi$. Assume that $H$ and $T$ are rotation invariant operators on $\mathcal{F}$. Then $F_{\chi}(H, T)$ is invariant under rotations. If there exists a $\underline{w} \in \mathcal{W}_{\xi}$ such that $F_{\chi}(H, T)=H[\underline{w}]$, then $H_{0,1}[\underline{w}]=H_{0,1}[\underline{w}]=0$.

Proof. (a). From Lemma 3.2 and the definition (3.12) it follows that $F_{\chi}\left(H_{g}-z, H_{0}-z\right)$ is rotation invariant. Thus the rotation invariance of $F_{z, g}$ now follows from Lemma 3.3. Hence by Theorem [2.3, $H_{0,1}\left[\underline{w}_{z, g}\right]=H_{0,1}\left[\underline{w}_{z, g}\right]=0$. (b). From Lemma 3.2 and the definition (3.12) it follows that $F_{\chi}(H, T)$ is rotation invariant. Hence by Theorem 2.3 , $H_{0,1}[\underline{w}]=H_{0,1}[\underline{w}]=0$.

Remark 2. Part (a) of the corollary is used for a so called initial Feshbach operator and Part (b) is used for each renormalization step. Corollary 3.4 is stated under the assumptions that the Feshbach operator can be expressed in terms of integral kernels (2.19). In [6] this assumption is verified for the initial step, see also [1]. For the renormalization step this property is shown to hold under the natural assumptions needed for operator theoretic renormalization, [2].

\section{A Appendix}

In this appendix we give a rigorous definition of (2.17), which does not involve creation or annihilation operators, and we provide a proof of (2.18). We introduce the Hilbert space $L_{s}^{2}\left(\left\{\mathbb{R}^{3} \times \mathbb{Z}_{2}\right\}^{n}\right)$ of complex valued square integrable functions which are totally symmetric with respect to the interchange of arguments belonging to different factors of the $n$-fold Cartesian product. We will identify this Hilbert space with a subspace of Fock space, by means of the canonical isomorphism of Hilbert spaces

$$
\mathcal{F}_{n} \cong L_{s}^{2}\left(\left\{\mathbb{R}^{3} \times \mathbb{Z}_{2}\right\}^{n}\right)
$$


Let $\varphi_{p} \in \mathcal{F}_{p}$ and $\psi_{q} \in \mathcal{F}_{q}$. If

$$
p-m=q-n \geq 0,
$$

we define

$$
\begin{aligned}
\left\langle\varphi_{p}, H_{m, n}[\underline{w}] \psi_{q}\right\rangle:= & \sqrt{\frac{q !}{l !} \sqrt{\frac{p !}{l !}}} \int_{X^{m} \times X^{l} \times X^{n}} d \hat{K}^{(l)} \frac{d \tilde{K}^{(m)}}{\left|\tilde{K}^{(m)}\right|^{1 / 2}} \frac{d K^{(n)}}{\left|K^{(n)}\right|^{1 / 2}} \\
& \times \varphi_{p}\left(\tilde{K}^{(m)}, \hat{K}^{(l)}\right) w_{m, n}\left(\Sigma\left[\hat{K}^{(l)}\right] ; \tilde{K}^{(m)}, K^{(n)}\right) \psi_{q}\left(K^{(n)}, \hat{K}^{(l)}\right),
\end{aligned}
$$

where we used the following definitions, $l:=p-m=q-n$. If (A.1) does not hold, we define

$$
\left\langle\varphi_{p}, H_{m, n}[\underline{w}] \psi_{q}\right\rangle:=0 .
$$

The goal of the remaining part of the Appendix is to show (2.18). It will then follow from (2.18) and the Riesz representation theorem, that $H_{m, n}[\underline{w}]$ defines a bounded operator on Fock space. In the case of (A.1) we estimate using Cauchy-Schwarz

$$
\begin{aligned}
& \left|\left\langle\varphi_{p}, H_{m, n}[\underline{w}] \psi_{q}\right\rangle\right| \leq \\
& \quad D_{l, p}\left(\varphi_{p}\right) D_{l, q}\left(\psi_{q}\right)\left\{\int_{X^{m} \times X^{n}}\left|K^{(n)}\right|^{-2}\left|\tilde{K}^{(m)}\right|^{-2} \sup _{r \in I}\left|w_{m, n}\left(r ; \tilde{K}^{(m)}, K^{(n)}\right)\right|^{2}\right\}^{1 / 2},(A)
\end{aligned}
$$

where we defined

$$
D_{l, p}\left(\varphi_{p}\right):=\sqrt{\frac{p !}{l !}}\left\{\int_{X^{m} \times X^{l}} d \tilde{K}^{(m)} d \hat{K}^{(l)}\left|\tilde{K}^{(m)}\right|\left|\varphi_{p}\left(\tilde{K}^{(m)}, \hat{K}^{(l)}\right) P_{\text {red }}\left(\tilde{K}^{(m)}, \hat{K}^{(l)}\right)\right|^{2}\right\}^{1 / 2},
$$

and inserted $P_{\text {red }}\left(\tilde{K}^{(m)}, \hat{K}^{(l)}\right):=1_{\Sigma\left[\tilde{K}^{(m)}\right]+\Sigma\left[\hat{K}^{(l)}\right] \leq 1}$ justified by (2.14). Now using the symmetry property of the wavefunctions, one finds

$$
\begin{aligned}
D_{l, p}\left(\varphi_{p}\right) & =\sqrt{\frac{p !}{l !}}\left\{\int_{X^{1} \times X^{m-1} \times X^{l}} d K^{(1)} d \tilde{K}^{(m-1)} d \hat{K}^{(l)}\left|K^{(1)}\right|\left|\tilde{K}^{(m-1)}\right|\right. \\
& \left.\times\left|\varphi_{p}\left(K^{(1)}, \tilde{K}^{(m-1)}, \hat{K}^{(l)}\right) P_{\mathrm{red}}\left(K^{(1)}, \tilde{K}^{(m-1)}, \hat{K}^{(l)}\right)\right|^{2}\right\}^{1 / 2} \\
& =\sqrt{\frac{p !}{(l+1) !}}\left\{\int_{X^{m-1} \times X^{l+1}} d \tilde{K}^{(m-1)} d \hat{K}^{(l+1)}\left|\tilde{K}^{(m-1)}\right|\right. \\
& \left.\times\left|\left(\Sigma\left[\hat{K}^{(l+1)}\right]\right)^{1 / 2} \varphi_{p}\left(\tilde{K}^{(m-1)}, \hat{K}^{(l+1)}\right) P_{\text {red }}\left(\tilde{K}^{(m-1)}, \tilde{K}^{(l+1)}\right)\right|^{2}\right\}^{1 / 2} \\
& \leq \sqrt{\frac{p !}{(l+1) !}}\left\{\int_{X^{m-1} \times X^{l+1}} d \tilde{K}^{(m-1)} d \hat{K}^{(l+1)}\left|\tilde{K}^{(m-1)}\right|\right. \\
& \left.\times\left|\left(H_{f}\right)^{1 / 2} \varphi_{p}\left(\tilde{K}^{(m-1)}, \hat{K}^{(l+1)}\right) P_{\text {red }}\left(\tilde{K}^{(m-1)}, \tilde{K}^{(l+1)}\right)\right|^{2}\right\}^{1 / 2} .
\end{aligned}
$$

Iterating above estimate we arrive at $D_{l, p}\left(\varphi_{p}\right) \leq\left\|H_{f}^{m / 2} 1_{H_{f} \leq 1} \varphi_{p}\right\|$. Inserting this into (A.4) gives

$$
\left|\left\langle\varphi_{p}, H_{m, n}[\underline{w}] \psi_{q}\right\rangle\right| \leq\left\|w_{m, n}\right\|_{\mathcal{W}_{m, n}}\left\|\varphi_{p}\right\|\left\|\psi_{q}\right\| .
$$


This together with (A.3) yields that for any two vectors $\varphi=\left(\varphi_{n}\right)_{n \in \mathbb{N}_{0}}$ and $\psi=\left(\psi_{n}\right)_{n \in \mathbb{N}_{0}}$ in $\mathcal{F}$ (i.e., $\varphi_{n}, \psi_{n} \in \mathcal{F}_{n}$ ) the following inequality holds,

$$
\left|\left\langle\varphi, H_{m, n}[\underline{w}] \psi\right\rangle\right| \leq\left\|w_{m, n}\right\|_{\mathcal{W}_{m, n}} \sum_{l=0}^{\infty}\left\|\varphi_{l+m}\right\|\left\|\psi_{l+n}\right\| \leq\left\|w_{m, n}\right\|_{\mathcal{W}_{m, n}}\|\varphi\|\|\psi\|,
$$

and hence (2.18) follows.

\section{References}

[1] V. Bach, J. Fröhlich, I.M. Sigal, Renormalization group analysis of spectral problems in quantum field theory, Adv. Math. 137 (1998), 205-298.

[2] V. Bach, T. Chen, J. Fröhlich, I.M. Sigal, Smooth Feshbach map and operatortheoretic renormalization group methods, J. Funct. Anal. 203 (2003), 44-92.

[3] M. Griesemer, D. Hasler, On the smooth Feshbach-Schur Map, J. Funct. Anal. 254 (2008), 2329-2335.

[4] D. Hasler, I. Herbst, On the self-adjointness and domain of Pauli-Fierz type Hamiltonians. Rev. Math. Phys. 20 (2008), 787-800.

[5] D. Hasler, I. Herbst, Ground states in the spin boson model, Ann. Henri Poincaré 12(4) (2011) .

[6] D. Hasler, I. Herbst, Convergent expansions in non-relativistic QED: Analyticity of the ground state, submitted. arXiv:1005.3522

[7] D. Hasler, I. Herbst, Smoothness and analyticity of perturbation expansions in QED, submitted. arXiv:1007.0969 